\title{
Computerized Physician Order Entry in the Neonatal Intensive Care Unit: A Narrative Review
}

\author{
Jaclyn B. York ${ }^{1}$ Megan Z. Cardoso ${ }^{1}$ Dara S. Azuma ${ }^{1} \quad$ Kristyn S. Beam ${ }^{1}$ Geoffrey G. Binney Jr. ${ }^{2}$ \\ Saul N. Weingart ${ }^{3}$
}

${ }^{1}$ Department of Pediatrics, Floating Hospital for Children at Tufts Medical Center, Boston, Massachusetts, United States

2 Department of Neonatal-Perinatal Medicine, Floating Hospital for Children at Tufts Medical Center, Boston, Massachusetts, United States

${ }^{3}$ Department of Medicine, Tufts Medical Center, Boston,

Massachusetts, United States

Address for correspondence Jaclyn B. York, MD, Department of Pediatrics, Floating Hospital for Children, Tufts Medical Center, 800 Washington Street, Boston, MA 02111, United States (e-mail: jyork@tuftsmedicalcenter.org).

Appl Clin Inform 2019;10:487-494.

Abstract

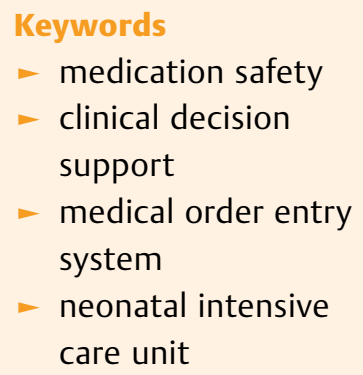

Background Computerized physician order entry (CPOE) has grown since the early 1990s. While many systems serve adult patients, systems for pediatric and neonatal populations have lagged. Adapting adult CPOE systems for pediatric use may require significant modifications to address complexities associated with pediatric care such as daily weight changes and small medication doses.

Objective This article aims to review the neonatal intensive care unit (NICU) CPOE literature to characterize trends in the introduction of this technology and to identify potential areas for further research.

Methods Articles pertaining to NICU CPOE were identified in MEDLINE using MeSH terms "medical order entry systems," "drug therapy," "intensive care unit, neonatal," "infant, newborn," etc. Two physician reviewers evaluated each article for inclusion and exclusion criteria. Consensus judgments were used to classify the articles into five categories: medication safety, usability/alerts, clinical practice, clinical decision Support (CDS), and implementation. Articles addressing pediatric (nonneonatal) CPOE were included if they were applicable to the NICU setting.

Results Sixty-nine articles were identified using MeSH search criteria. Twenty-two additional articles were identified by hand-searching bibliographies and 6 articles were added after the review process. Fifty-five articles met exclusion criteria, for a final set of 42 articles. Medication safety was the focus of 22 articles, followed by clinical practice (10), CDS (10), implementation (11), and usability/alerts (4). Several addressed more than one category. No study showed a decrease in medication safety post-CPOE implementation. Within clinical practice articles, CPOE implementation showed no effect on blood glucose levels or time to antibiotic administration but showed conflicting results on mortality rates. Implementation studies were largely descriptive of single-hospital experiences.

Conclusion CPOE implementation within the NICU has demonstrated improvement in medication safety, with the most consistent benefit involving a reduction in medication errors and wrong-time administration errors. Additional research is needed to understand the potential limitations of CPOE systems in neonatal intensive care and how CPOE affects mortality. received

October 5, 2018

accepted after revision

April 30, 2019 (c) 2019 Georg Thieme Verlag KG Stuttgart · New York
DOI https://doi.org/

10.1055/s-0039-1692475. ISSN 1869-0327. 


\section{Background and Significance}

The Federal government, National Academy of Medicine (formerly Institute of Medicine), and the Leapfrog Group all endorse the use of information technology in health care to decrease medical errors. ${ }^{1}$ Computerized physician/provider order entry (CPOE) is one important information technology that has demonstrated the ability to prevent medication errors within the hospital. ${ }^{2}$ While many CPOE systems serve adult patients, development of systems for pediatric and neonatal inpatients have lagged. ${ }^{3}$ Delayed implementation of pediatric CPOE may reflect the additional complexities associated with pediatric care. Such as (1) frequent weight changes and the need for precise measurement (e.g., weights in the NICU population are often measured down to the gram), (2) high-risk medications, (3) small medication doses, (4) immunizations, and (5) pediatric data capture (e.g., the calculation of postmenstrual age and gestational age in neonates). ${ }^{4,5}$ The neonatal population is especially vulnerable and there is evidence that medication errors occur more often in the neonatal intensive care unit (NICU) than anywhere else in the hospital. ${ }^{6}$

The goal of this narrative review is to examine the research literature addressing NICU CPOE in order to assess the impact of this technology on patient safety (specifically, medication errors) and implementation efforts, and to identify areas for further research.

\section{Methods}

\section{Search Strategy}

Articles related to NICU CPOE were identified in MEDLINE using a combination of MeSH terms including "medical order entry systems," "drug therapy," "computer assisted," "intensive care unit, pediatric" and "intensive care unit, neonatal," and "infant, newborn." We included articles published in English in the past 30 years. The final search was run on April 4, 2016. Articles were then added as recommended by the reviewers during the revision process.

\section{Study Selection}

Four physicians (D.A., J.B.Y., K.S.B., and M.C.) reviewed each article for inclusion in the review. Titles and abstracts were reviewed initially for appropriateness. Full texts were then evaluated for inclusion and exclusion criteria which included the keywords listed earlier and English-language publication. We excluded articles that lacked reference to weight-based dosing or CPOE implementation. Bibliographies were then hand searched for additional articles that met inclusion criteria. Consensus judgment by all four reviewers was used to classify the articles into five categories: medication safety, usability/alerts, clinical practice, clinical decision support (CDS), and implementation. Articles addressing pediatric (nonneonatal) CPOE were included if they were applicable to the NICU setting (e.g., using weight-based dosing or high-risk medications). Articles were then classified according to the primary issue(s) addressed. There was no abstraction tool used due to the heterogeneity of study populations and methods.

\section{Category Definition}

Medication safety. Articles were classified as describing medication safety if there was an evaluation of the incidence of medication errors associated with implementation of $\mathrm{CPOE}$, or if there was a before-and-after evaluation of adverse medication events.

Clinical practice. Clinical practice articles described the impact of CPOE on day-to-day activity in a critical care environment after implementation of CPOE.

CDS. CDS articles described the implementation and evaluation of a CDS tool as part of the CPOE system.

Implementation. Implementation articles described experiences related to the introduction of a CPOE system into a healthcare setting.

Usability/Alerts. Usability/Alerts articles dealt specifically with daily utilization of a CPOE system including frequent alert pop-ups.

\section{Results}

We identified 69 articles using MeSH search criteria. Twentytwo additional articles were identified by hand-searching bibliographies and 6 articles were added during the revision process based on reviewer's recommendations. Of those 97 articles 55 were excluded, yielding a total of 42 articles included in the review ( - Fig. 1). Medication safety was the focus of 22 articles, ${ }^{6-27}$ followed by clinical practice (10 articles), ${ }^{18,28-36}$ CDS (10 articles), $6,11,12,20,25,29,35-38$ implementation (11 articles), $22,28-30,34,39-44$ and usability/alerts (4 articles). ${ }^{12,45-47}$ Twenty articles addressed two categories and only one article addressed three categories (-Fig. 2).

\section{Medication Safety}

Of the 22 articles that addressed medication safety, none demonstrated an increase in medication safety errors postCPOE implementation. Four of the studies were systematic reviews that examined medication safety at the time of CPOE implementation. ${ }^{7,9,22,26}$ One study determined a baseline prevalence of medication errors prior to $\mathrm{CPOE}^{7}$ and the other three studies found medication error reduction after CPOE implementation. ${ }^{9,22,26}$ of the remaining 18 primary studies, 6 found that CPOE implementation produced a reduction in medication errors $8,11,12,18,19,25$ and 3 showed a decrease in the opportunity for error. ${ }^{10,13,19}$ On the other hand, one study illustrated new potential errors that coincided with implementing CPOE. ${ }^{13}$

One study found no significant reduction in medication dosing error rates before and after $\mathrm{CPOE}$ implementation, but did find a significant reduction in error rates after CDS was implemented (54-34\%, $p<0.001$ ). ${ }^{12}$ Horri and colleagues reported that medication errors in two different French University NICUs increased when an order was hand written instead of being ordered by CPOE. Additional pre- and postevaluation of their CPOE system implementation found a significant reduction in opportunity for error with an absolute risk reduction of $8 \%{ }^{8}$ Kaushal and colleagues studied 1,120 patients at two academic hospitals and reported that rates of potential adverse drug events were higher in the 


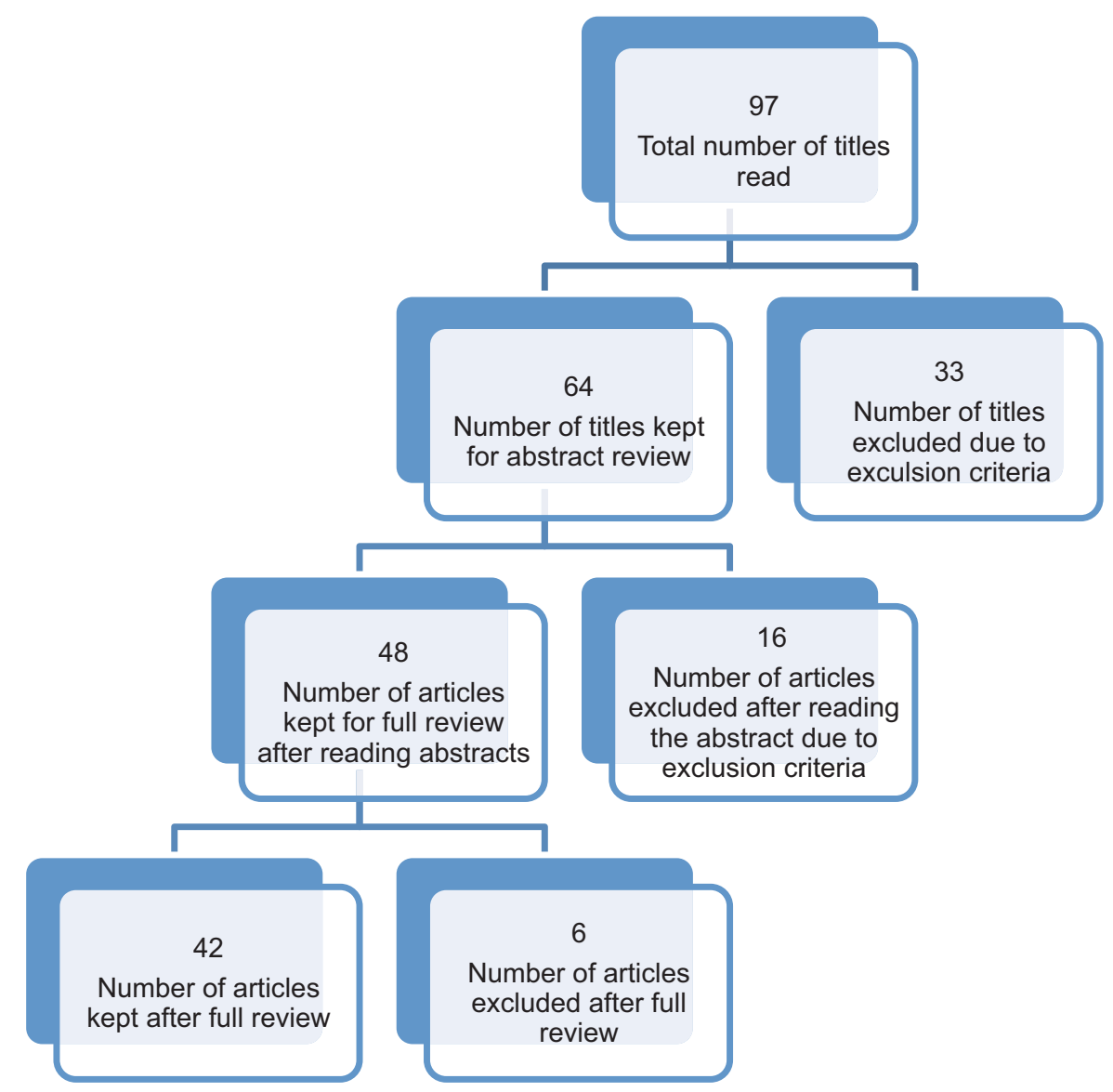

Fig. 1 Article tree delineating which articles were selected for review.

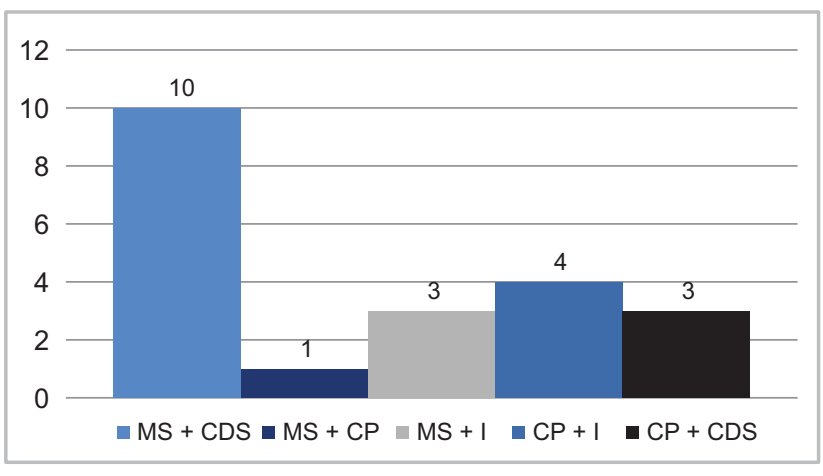

Fig. 2 Articles addressing more than one category. MS, medication safety; CDS, clinical decision support; CP, clinical practice; I, implementation.

neonatal population than in adults (79\% at the stage of drug ordering). ${ }^{11}$ After establishing that the potential for error was greater in the neonatal population, Kaushal and colleagues then reviewed 12 trials evaluating the effects of $\mathrm{CPOE}$ and CDS on medication safety in the NICU. They found that 5 of the 12 studies showed a significant decrease in medication error rates after CPOE introduction in the NICU. ${ }^{12}$ Walsh and colleagues studied the NICU, pediatric intensive care unit (PICU), and pediatric inpatient units and found no significant change in unrecognized medication errors before and after CPOE implementation. ${ }^{23}$ In their meta-analysis of 12 studies, van Rosse and colleagues showed that there was a statistically significant decrease in medication prescription errors with CPOE. ${ }^{22}$ Chedoe and colleagues observed that there is no consensus on how to define medication errors in the NICU population, making identification of these events difficult. In addition, heterogeneity in reporting of adverse events makes it difficult to define the type and frequency of NICU medication errors. ${ }^{7}$ Chuo and Hicks reviewed NICU medication errors from 2001 to 2005 from the MEDMARX voluntary medication error reporting program. They found an apparent reduction in the percentage of errors that reached the patient using CPOE versus older computer order entry systems. Additionally, there were fewer transcription errors with CPOE. While there was a reduction in wrong dose and wrong time errors, there was an increase in the wrong dosage form (e.g., liquid vs. tablet) ${ }^{26} \mathrm{~A}$ natural language processing algorithm, validated by Temple and colleagues ${ }^{48}$ to predict NICU discharges, was used at Cincinnati Children's Hospital Medical Center to automatically detect medication errors within the NICU. Once this system was implemented, the algorithm's sensitivity and specificity for identification of errors was similar to manual identification. ${ }^{15}$

Jozefczyk and colleagues used 18 predefined criteria created from the Joint Commission medication use standards. Opportunity for error was defined as "the increased likelihood of a medication error based on adherence to the 
listed criteria. A medication order has the greatest opportunity for correct medication use when all 18 of the listed criteria are present or performed." ${ }^{9}$ In this article, the researchers demonstrated that the number of orders with zero opportunity for error greatly increased after CPOE was introduced, from 42 to $98 \%(p<0.001)$.

Another research team found reduced variation in medication administration after CPOE implementation. ${ }^{21}$ In this study, variation referred to a discrepancy between the order and the medication administration. Causes of variation included availability of the medication, order needing clarification, another patient needing immediate attention, or miscommunication between nurses and pharmacy or physician. Variation occurred when a different medication was given than was ordered, wrong time of administration, different dosage, and different mode of delivery than ordered. The greatest area of variation involved wrong time of administration, which was reduced in the post-CPOE time period from $53.1 \%$ of all variances to $6.7 \%$ of all variances. ${ }^{21}$

When using a quality improvement cycle after CPOE implementation, Myers and colleagues saw no difference in birth weight-specific survival rates, but they did see a reduction in neonatal medication errors from 3.2 to 0.6 errors per 1,000 patient-days, which was associated with a substantial decrease in average total hospital cost per infant. They also noted that average length of stay was decreased for infants whose birth weights were less than 1,001 g. $^{18}$

Two articles examined potential errors in medication administration. Chappell and Newman studied potential errors with the small doses prescribed for neonates. ${ }^{27}$ They examined 336 intravenous medication orders for 1,348 intravenous drug doses. In this study, investigators found that doses would be less than one-tenth of the vial, which could increase the risk of a substitution overdose. In total, $104(31 \%)$ of the prescriptions or $333(25 \%)$ of doses administered had the potential for a 10 -fold overdose. ${ }^{27}$ Koppel and colleagues found that a widely used CPOE system facilitated 22 types of medication error risks. ${ }^{13}$ Examples included fragmented CPOE displays that prevent a comprehensive view of patients' medications, pharmacy inventory displays mistaken for dosage guidelines, ignored antibiotic renewal notices placed on paper charts rather than in the CPOE system, separation of functions that facilitate double dosing and incompatible orders, and inflexible ordering formats generating incorrect orders.

Overall, the 23 studies examining medication safety showed that implementation of a CPOE system would either decrease or maintain the same rate of medication errors in critical care environments including the NICU. The studies done by Walsh et $\mathrm{al}^{23}$ and Wang et $\mathrm{al}^{24}$ looked at medication errors with a large sample size and rigorous inclusion criteria; however, none of these studies looked at whether or not the medication errors resulted in a clinically significant error. Also, most of the articles reviewed were conducted at academic centers making it difficult to generalize their results to community centers (-Supplementary Appendix A, available in the online version).

\section{Clinical Practice}

Ten articles described the impact of $\mathrm{CPOE}$ on various aspects of clinical practice. Maat and colleagues found no difference in the degree of hypo- or hyperglycemia before and after CPOE implementation. ${ }^{35}$ However, they found that CDS reduced the time required for both simple and complex calculations. Chapman and colleagues found that CPOE reduced the time from NICU admission to pharmacy verification, but did not reduce the time to antibiotic administration. ${ }^{4}$ Another study by Cordero et al found a reduction in time to caffeine administration in the NICU and an overall benefit of CPOE in terms of reduction in medication administration times and medication errors for specific drugs. Radiological services were ordered and performed in a more timely manner as well. ${ }^{29}$ Myers et al found that implementation of a CPOE system changed clinical practices in different domains including pharmacy, laboratory, respiratory therapy, and radiology. An interactive physician order entry system was used to promote quality improvement and cost-effective care as noted earlier. However, this study also looked at birthweight-specific survival rates before and after CPOE implementation which did not show any change. The reduction in medical errors was cost-effective by improving outcomes (e.g., decreased length of stay) but did not improve mortality. ${ }^{18}$

\section{Clinical Decision Support}

Studies in the adult population have found significant variation in the use of decision support to detect and intervene when medication orders would result in serious harm to a patient. The use of advanced decision support tools was suggested as a way to prevent serious errors. ${ }^{38}$ The pediatric literature has shown promising results in the setting of pediatric-specific interventions. Nine studies examined CDS tools as part of a CPOE system. Kazemi et al reported improvement when they examined a step-wise implementation of both CPOE and a CDS tool. ${ }^{12}$ They found that there was no statistically significant difference in medication errors after the introduction of CPOE without CDS functionality, 52 to $50 \%$, respectively. However, after the implementation of a CDS system, medication errors fell to 33\%. Their CDS system generated alerts and warnings that were largely ignored when physicians did not understand the warnings. For example, when the CDS alerted the resident that a prescribed dose was incorrect due to recalculated glomerular filtration rate, residents often ignored these alerts and assumed the alerts were incorrect. The authors suggested that inclusion of an explanation of the warnings would improve compliance. ${ }^{12}$ Clinicians may find an alert more compelling if the alert displays the calculations for a suggested dose instead of presenting the dose alone. Other factors include alert fatigue, physician resistance, or inappropriate alerts. Finally, a study looking at 840 patient visits reported a reduction from 24 errors per 100 visits to 13 errors per 100 visits after a quicklist (a CDS tool to assist with drug dosing of the most commonly utilized medications) was added to CPOE. This quicklist was used for approximately $30 \%$ of the medication orders placed during these visits. 
Looking at orders placed exclusively with the quicklist, there were 1.87 errors per 100 orders compared to 18.28 errors per 100 orders when the quicklist was not used. ${ }^{20}$ In general, the researchers saw the most effective reduction in medication errors when CDS augmented the implementation of CPOE.

\section{Implementation}

Eleven articles assessed practices related to implementation of a CPOE system. Han and colleagues reported a significant increase in mortality from 2.8 to $6.5 \%$ after the implementation of $\mathrm{CPOE}^{30} \mathrm{~A}$ further investigation into this result found a change in established processes that led to certain medications no longer being immediately available on the intensive care unit. Medications were moved to the central pharmacy leading to disruptions in work flow. Additional analysis revealed that the children who did not survive were overall more seriously ill than those who died during the initial 3-month study period. Another study conducted in response to the Han et al's article by Sittig and colleagues examined the potential for this increase in mortality. ${ }^{34}$ They found that the primary reason CPOE implementation failed was due to a disruption in patient care workflow. They proposed that CPOE implementation should be done over a 1- to 3-year period to allow people to adapt slowly to changes in work flow. They also recommended mock drills prior to implementation to identify unexpected problems. Keene and colleagues did not find increased mortality among similarly ill patients after CPOE implementation in the NICU. ${ }^{31}$ The authors suggested that a longer, 2-year development period prior to implementation ensured safe rollout, leading to no change in mortality.

Giannone described challenges associated with CPOE implementation in a hospital system, including a lack of focus or attention to specific populations. As NICU beds accounted for only $6 \%$ of the total inpatient beds in the hospital system, there was less of an institutional focus on this small population. Meticulous fluid management of lowbirth-weight infants requires specific considerations that are not always built into CPOE systems meant for adults. ${ }^{43}$

A study by Beam et al assessed perceptions of pre- and postimplementation of CPOE in a single-center NICU. ${ }^{44}$ Both physicians and nurses reported a dramatic increase in job satisfaction, despite preimplementation concerns that training was not sufficient and that learning would be difficult. There were differences among professions regarding concerns about a potential adverse impact; nurses were more concerned than physicians in the postimplementation phase of the study.

Many articles describe a multidisciplinary approach to CPOE development including input from nurses, physicians, pharmacists, respiratory therapist, and unit clerks, who often have a good insight into the workflow of the unit. These articles suggest that the clinical team should work in concert with the information and technology team to customize CPOE for pediatric populations. A common theme is the difficulty of trying to adapt adult CPOE to meet the needs of the pediatric population, especially a NICU population. Several articles also pointed out that creating order sets can improve user satisfaction. ${ }^{40-43}$

\section{Usability and Alerts}

Four articles addressed CPOE usability. One article described a single institution's creation of a personalized prescription feedback system. Developed through several quality improvement cycles, the system sent emails to individual clinicians when there was a prescription medication error in a nonpunitive fashion with a goal to improve overall medication safety. The authors found a reduction in narcotic prescription by $83 \%$ as well as an increase from 3.94 to 22.63 days between narcotic prescription errors after implementation of this personalized prescription feedback system to clinicians. ${ }^{46}$ Another article by Sheehan et al examined the types and frequency of alerts in the CPOE system within the context of antibiotic orders and found that most alerts addressed critical lab information and patient factors that may lead to adverse events with the drug administration. They also found that the alerts were targeted at the appropriate health care providers. ${ }^{45}$ As mentioned in the CDS section, Kazemi and colleagues found through interviews and personal discussions that alerts were often ignored if the provider did not understand them, had alert fatigue, or if the alert was inappropriate. ${ }^{12}$ Finally, primary care considerations are not often addressed in the ICU setting. Ernst looked at the effects of an alert to remind clinicians to give 2-month vaccines to neonates older than 58 days. This alert improved immunization rates in the NICU from 71 to $94 \%$ $(p<0.0001) .{ }^{47}$ The alert also decreased the vaccination time from day 71 of life to day 64 of life $(p<0.0001)$.

\section{Discussion}

In this narrative review, we found that multiple studies documented an increase in patient safety with the implementation of CPOE systems and an increased benefit with the implementation of CDS. Mortality rates did not definitively improve, but this could reflect differences in implementation, strategic planning, and the effectiveness of the IT implementation teams. CPOE introduction may lead to detrimental effects, especially in sites with rapid development and implementation. ${ }^{30,34}$ Recognizing the specific needs of the NICU population, Chuo and Hicks recommended evaluating emerging technology in terms of five categories: (1) context which determines the needs and workflow of the unit, (2) unit attributes including statistics and culture of the NICU, (3) personnel and role identification (e.g., house staff, attending, pharmacist, nurse), (4) technology performance (including a comparative analysis of how it will work in the specific NICU), and (5) health policy perspective (comparing the money spent on technology vs. the cost of patient safety). ${ }^{26}$

All studies described the implementation of commercial CPOE products, typically developed for use in an adult population, that were subsequently adapted for use in the NICU. The NICU patients are particularly complex and face challenges not found in adult or older pediatric populations. Beam et a ${ }^{44}$ described a multitude of considerations that had to be taken into consideration during the development stage of their NICU CPOE product and included decision support 
for over 200 medications (many of which were off-label), current weight, gestational age, postmenstrual age, and work flow for verifying orders. They developed a weekly weight "re-write" policy to help ensure appropriate dosing given frequent weight changes in the NICU. ${ }^{44}$ Additionally, physicians must consider fetal physiology and the frequent use of loading and maintenance dosing (e.g., caffeine and phenobarbital). Long-term dependence on parenteral nutrition and other specialized parenteral fluids present additional challenges. The articles included in this narrative review provided strategies that discussed the management of frequent dosing weight changes, timing and management of loading and maintenance dosing, and/or long-term nutrition management. Such strategies include having quicklists or order sets to choose from with neonatal-specific dosing and alerts generated when the order was not a standard dose for neonates. As long as the physicians understood what the alerts meant, these were functional strategies for reducing medication errors. The authors believe that these are areas of great importance as CPOE systems are implemented and adapted to NICUs.

The majority of studies examining CPOE in the NICU examine medication safety. While this remains a very important aspect of CPOE implementation, there is opportunity to conduct research on other aspects of CPOE in neonatology. One example includes the complex calculations and high risks involved with total parenteral nutrition which were not examined in this review. In addition, changes in pharmacy processes and procedures such as time to first antibiotic dose or time to first glucose measurement should be examined, as these are equally critical in the initial management of a neonate in the intensive care unit. Articles that addressed the addition of pharmacists noted that the pharmacist often intercepted physician order entry errors but missed errors in medication administration.

The frequency and heterogeneity of alerts used in CPOE was also noted to be an issue. The types of alerts may not be specific to the NICU and or to individual patients. These alerts do not routinely take into account NICU-specific dosing principles including postgestational age, frequent bolus dosing, and frequent medication dose changes. When the physicians do not understand what these alerts are relating to, they are often ignored and may result in poor outcomes. These alerts should be minimal in frequency to avoid alert fatigue when inputting orders into a system.

The heterogeneity of the articles' methods likely contributed to the variability in the authors' conclusions. Implementation strategies described in the articles varied considerably. However, slow implementation and integration into workflow appeared to offer a softer and less disruptive approach.

There are several limitations of this narrative review. First, a systematic review was not deemed to be possible, as there is a great deal of heterogeneity in primary outcomes as well as interventions assessed relating to different CPOE systems. Many of the studies examined both PICU and NICU. In addition to being critically ill, NICU patients are particularly susceptible to errors given that NICUs tend to have a more homogenous population, which may lead to more wrong patient medication administrations than PICUs. Including PICUs in this review may make some of the conclusions difficult to interpret for NICUs. Second, NICUs reported medication errors in many different ways. Some relied on incident reporting and some relied on retrospective chart review. This led to differences in the baseline error rates between units and limits generalizability. In future studies, researchers could use more uniform or standardized methods. Strengths of this narrative review include a two-person evaluation of each article. To the best of our knowledge, we included all of the articles evaluating implementation and discussion of CPOE in an NICU.

In conducting our search for articles discussing $\mathrm{CPOE}$, it became clear that there is a paucity of literature on this topic. We postulate that this could be due to several factors including the vast number of differing CPOE systems and the relatively recent time in which they have been implemented on a large scale. Given these factors, we did opt to include several descriptive articles even though they are not as easily replicated as the larger multicenter studies. We were able to look at two articles derived from large multicenter trials which were conducted with an effort to minimize bias to produce objective results, and there were several other studies looking at large academic centers that could be replicated in order to make the results more generalizable.

\section{Conclusion}

CPOE is mandated by government and regulatory agencies as the safest way to prescribe medications with the most consistent benefit involving medication errors and wrongtime administration errors ( - Fig. 3). However, research has demonstrated that CPOE implementation within the NICU has demonstrated equivocal improvement in mortality. This may be due to the fact that the NICU population, with its unique clinical characteristics, may not be receiving the same safety benefits as the adult medical population. As noted in the articles mentioned earlier, many institutions have developed or adapted individual systems with the goal of providing safer CPOE systems, but there has not been a consistent standardized approach identified to prevent medication errors in this fragile population. These authors believe that

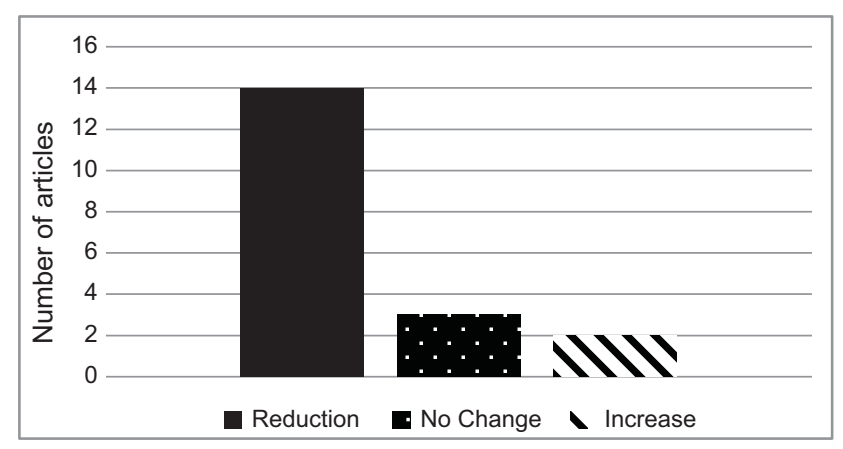

Fig. 3 Aggregate number of articles showing reduction, no change, or increase in medication safety errors after the implementation of computerized physician order entry. 
standardizing CPOE systems to account for the NICU population's needs and conducting vigorous research in order to prove effectiveness is essential to providing optimal care. Steps also need to be initiated in order to develop neonatespecific products which could reduce potential errors. Additional research to assess the impact of errors on morbidity, mortality, and hospital length of stay is needed in order to understand the value and potential limitations of CPOE systems in neonatal intensive care.

\section{Clinical Relevance Statement}

This review provides a succinct, comprehensive overview of how the implementation of CPOE has affected the NICU. The authors found an overall decrease in medication safety errors and an additional benefit when CDSs were in place. However, the NICU is a unique patient population and as such the successful implementation of CPOE required specialized order sets and frequent medication dosing adjustments.

\section{Multiple Choice Questions}

1. Research has shown that variation in medication administration decreased after CPOE implementation. Which of the following is an area of medication administration variation noted in the earlier review?
a. Multiple orders per patient.
b. Wrong time of administration.
c. Miscommunication between staff.
d. Medication given to wrong patient.

Correct Answer: The correct answer is option b. The areas of variation involved were different medication given than was ordered, wrong time of administration, different dosage, and different mode of delivery than ordered. The greatest area of variation involved wrong time of administration, which was reduced in the post-CPOE time period from $53.1 \%$ of all variances to $6.7 \%$ of all variances. This is different from the causes of the variation which included availability of the medication, order needing clarification, another patient needing immediate attention, or miscommunication between nurses and pharmacy or physician.

2. Implementation of $\mathrm{CPOE}$ in the NICU has demonstrated the most consistent benefit in which of the following areas:

a. Medication errors.

b. Time to medication administration.

c. Infant mortality.

d. Hypoglycemia.

Correct Answer: The correct answer is option a. CPOE implementation within the NICU has demonstrated equivocal improvement in patient safety, with the most consistent benefit involving medication errors. Maat and colleagues found no difference in the degree of hypo- or hyperglycemia before and after CPOE implementation. Chapman et al found that CPOE reduced the time from NICU admission to pharmacy verification, but did not reduce the time to antibiotic administration.
Conflict of Interest

None declared.

\section{References}

1 Poon EG, Blumenthal D, Jaggi T, Honour MM, Bates DW, Kaushal R. Overcoming barriers to adopting and implementing computerized physician order entry systems in U.S. hospitals. Health Aff (Millwood) 2004;23(04):184-190

2 Bates DW, Leape LL, Cullen DJ, et al. Effect of computerized physician order entry and a team intervention on prevention of serious medication errors. JAMA 1998;280(15):1311-1316

3 Kim GR, Miller MR, Ardolino MA, Smith JE, Lee DC, Lehmann CU. Capture and classification of problems during CPOE deployment in an academic pediatric center. AMIA Annu Symp Proc 2007; $11: 414-417$

4 Chapman AK, Lehmann CU, Donohue PK, Aucott SW. Implementation of computerized provider order entry in a neonatal intensive care unit: Impact on admission workflow. Int J Med Inform 2012;81(05):291-295

5 Spooner SA; Council on Clinical Information Technology, American Academy of Pediatrics. Special requirements of electronic health record systems in pediatrics. Pediatrics 2007;119(03): 631-637

6 Donze A, Wolf M. Safety in the NICU: preventing medication errors with computerized provider order entry. Nurs Womens Health 2007;11(06):612-617

7 Chedoe I, Molendijk HA, Dittrich ST, et al. Incidence and nature of medication errors in neonatal intensive care with strategies to improve safety: a review of the current literature. Drug Saf 2007; 30(06):503-513

8 Horri J, Cransac A, Quantin C, et al. Frequency of dosage prescribing medication errors associated with manual prescriptions for very preterm infants. J Clin Pharm Ther 2014;39(06):637-641

9 Jozefczyk KG, Kennedy WK, Lin MJ, et al. Computerized prescriber order entry and opportunities for medication errors: comparison to tradition paper-based order entry. J Pharm Pract 2013;26(04): 434-437

10 Kaushal R, Bates DW, Landrigan C, et al. Medication errors and adverse drug events in pediatric inpatients. JAMA 2001;285(16): 2114-2120

11 Kaushal R, Shojania KG, Bates DW. Effects of computerized physician order entry and clinical decision support systems on medication safety: a systematic review. Arch Intern Med 2003; 163(12):1409-1416

12 Kazemi A, Ellenius J, Pourasghar F, et al. The effect of computerized physician order entry and decision support system on medication errors in the neonatal ward: experiences from an Iranian teaching hospital. J Med Syst 2011;35(01):25-37

13 Koppel R, Metlay JP, Cohen A, et al. Role of computerized physician order entry systems in facilitating medication errors. JAMA 2005; 293(10):1197-1203

14 Lefrak L. Moving toward safer practice: reducing medication errors in neonatal care. J Perinat Neonatal Nurs 2002;16(02): $73-84$

15 Li Q, Kirkendall ES, Hall ES, et al. Automated detection of medication administration errors in neonatal intensive care. J Biomed Inform 2015;57:124-133

16 Lillis K. Automated dosing. Computerized physician order entry reduces risk of medication and dosing errors in neonatal ICU. Health Manag Technol 2003;24(11):36-37

17 Lucas AJ. Improving medication safety in a neonatal intensive care unit. Am J Health Syst Pharm 2004;61(01):33-37

18 Myers TF, Venable HH, Hansen JA; NICU Clinical Effectiveness Task Force. Computer-enhanced neonatology practice evolution in an academic medical center. J Perinatol 1998;18(6, Pt 2, Suppl): S38-S44 
19 Potts AL, Barr FE, Gregory DF, Wright L, Patel NR. Computerized physician order entry and medication errors in a pediatric critical care unit. Pediatrics 2004;113(1, Pt 1):59-63

20 Sard BE, Walsh KE, Doros G, Hannon M, Moschetti W, Bauchner H. Retrospective evaluation of a computerized physician order entry adaptation to prevent prescribing errors in a pediatric emergency department. Pediatrics 2008;122(04):782-787

21 Taylor JA, Loan LA, Kamara J, Blackburn S, Whitney D. Medication administration variances before and after implementation of computerized physician order entry in a neonatal intensive care unit. Pediatrics 2008;121(01):123-128

22 van Rosse F, Maat B, Rademaker CM, van Vught AJ, Egberts AC, Bollen CW.The effect of computerized physician order entry on medication prescription errors and clinical outcome in pediatric and intensive care: a systematic review. Pediatrics 2009;123(04):1184-1190

23 Walsh KE, Landrigan CP, Adams WG, et al. Effect of computer order entry on prevention of serious medication errors in hospitalized children. Pediatrics 2008;121(03):e421-e427

24 Wang JK, Herzog NS, Kaushal R, Park C, Mochizuki C, Weingarten SR. Prevention of pediatric medication errors by hospital pharmacists and the potential benefit of computerized physician order entry. Pediatrics 2007;119(01):e77-e85

25 Yamamoto L, Kanemori J. Comparing errors in ED computerassisted vs conventional pediatric drug dosing and administration. Am J Emerg Med 2010;28(05):588-592

26 Chuo J, Hicks RW. Computer-related medication errors in neonatal intensive care units. Clin Perinatol 2008;35(01):119-139

27 Chappell K, Newman C. Potential tenfold drug overdoses on a neonatal unit. Arch Dis Child Fetal Neonatal Ed 2004;89(06):F483-F484

28 Castellanos I, Rellensmann G, Scharf J, Bürkle T. Computerized physician order entry (CPOE) in pediatric and neonatal intensive care: recommendations how to meet clinical requirements. Appl Clin Inform 2012;3(01):64-79

29 Cordero L, Kuehn L, Kumar RR, Mekhjian HS. Impact of computerized physician order entry on clinical practice in a newborn intensive care unit. J Perinatol 2004;24(02):88-93

30 Han YY, Carcillo JA, Venkataraman ST, et al. Unexpected increased mortality after implementation of a commercially sold computerized physician order entry system. Pediatrics 2005;116(06): 1506-1512

31 Keene A, Ashton L, Shure D, Napoleone D, Katyal C, Bellin E. Mortality before and after initiation of a computerized physician order entry system in a critically ill pediatric population. Pediatr Crit Care Med 2007;8(03):268-271

32 Longhurst CA, Parast L, Sandborg CI, et al. Decrease in hospitalwide mortality rate after implementation of a commercially sold computerized physician order entry system. Pediatrics 2010;126 (01):14-21

33 Maslove DM, Rizk N, Lowe HJ. Computerized physician order entry in the critical care environment: a review of current literature. J Intensive Care Med 2011;26(03):165-171
34 Sittig DF, Ash JS, Zhang J, Osheroff JA, Shabot MM. Lessons from "Unexpected increased mortality after implementation of a commercially sold computerized physician order entry system". Pediatrics 2006;118(02):797-801

35 Maat B, Rademaker CM, Oostveen MI, Krediet TG, Egberts TC, Bollen CW. The effect of a computerized prescribing and calculating system on hypo- and hyperglycemias and on prescribing time efficiency in neonatal intensive care patients. JPEN J Parenter Enteral Nutr 2013;37(01):85-91

36 Hum RS, Cato K, Sheehan B, et al. Developing clinical decision support within a commercial electronic health record system to improve antimicrobial prescribing in the neonatal ICU. Appl Clin Inform 2014;5(02):368-387

37 Sheehan B, Kaufman D, Stetson P, Currie LM. Cognitive analysis of decision support for antibiotic prescribing at the point of ordering in a neonatal intensive care unit. AMIA Annu Symp Proc 2009; 2009:584-588

38 Metzger J, Welebob E, Bates DW, Lipsitz S, Classen DC. Mixed results in the safety performance of computerized physician order entry. Health Aff (Millwood) 2010;29(04):655-663

39 Palma JP, Sharek PJ, Classen DC, Longhurst CA. neonatal informatics: computerized physician order entry. Neoreviews 2011; 12:393-396

40 Ramirez A, Carlson D, Estes C. Computerized physician order entry: lessons learned from the trenches. Neonatal Netw 2010; 29(04):235-241

41 Ventura ML, Battan AM, Zorloni C, et al. The electronic medical record: pros and cons. J Matern Fetal Neonatal Med 2011;24 (Suppl 1):163-166

42 Waitman LR, Pearson D, Hargrove FR, et al. Enhancing computerized provider order entry (CPOE) for neonatal intensive care. AMIA Annu Symp Proc 2003:1078

43 Giannone G. Computer-supported weight-based drug infusion concentrations in the neonatal intensive care unit. Comput Inform Nurs 2005;23(02):100-105

44 Beam KS, Cardoso M, Sweeney M, Binney G, Weingart SN. Examining perceptions of computerized physician order entry in a neonatal intensive care unit. Appl Clin Inform 2017;8(02): 337-347

45 Sheehan B, Chused A, Graham PL III, Stetson P, Currie L. Frequency and types of alerts for antibiotic prescribing in a neonatal ICU. Stud Health Technol Inform 2009;146:521-525

46 Sullivan KM, Suh S, Monk H, Chuo J. Personalised performance feedback reduces narcotic prescription errors in a NICU. BMJ Qual Saf 2013;22(03):256-262

47 Ernst KD. Electronic alerts improve immunization rates in twomonth-old premature infants hospitalized in the neonatal intensive care unit. Appl Clin Inform 2017;8(01):206-213

48 Temple MW, Lehmann CU, Fabbri D. Natural language processing for cohort discovery in a discharge prediction model for the neonatal ICU. Appl Clin Inform 2016;7(01):101-115 